\title{
ASSESSMENT WITHIN PHYSICAL EDUCATION TEACHER EDUCATION: THE CASE OF FONTYS UNIVERSITY IN THE NETHERLANDS
}

Evauación en la formación del Profesorado de educación física: en caso de la Fontys University en Holanda

Avaliação na formação de professores de educação física: o caso da Fontys University na Holanda

\author{
Gwen Weeldenburg (1)
}

Menno Slingerland (2)

(1) Fontys University of Applied Sciences, School of Sports Studies, Eindhoven, the Netherlands. Teléfono: +31 885073708. Correo electrónico: g.weeldenburg@fontys.nl

(2) Fontys University of Applied Sciences, School of Sports Studies, Eindhoven, the Netherlands. Teléfono: +31 885074071. Correo electrónico: m.slingerland@,fontys.nl

\begin{abstract}
In recent years, the Physical Education Teacher Education (PETE) curriculum of Fontys University has been completely redesigned (see our abstract in this monograph, round tables section). During this particular workshop we will present more background and detail on how we prepare our PETE students to become successful PE teachers. We will elaborate on the educational and assessment approach within our PETE program and discuss examples of learning tasks that we employ within our program to educate our students. We will present specific examples of assessment programs (i.e. school placement, movement domains, theory), authentic (formative and summative) assessment tasks and (digital) assessment tools. During the workshop, and in order to learn from each other, we invite you to discuss our approach to PETE and to share and compare your own experiences.
\end{abstract}

Keywords: Physical education teacher education; 4C/ID model; learning tasks; assessment;

programmatic assessment

\section{Resumen}

En los últimos años, el currículo para la formación inicial del profesorado de educación física (FIPEF) de la Fontys University ha sido rediseñado completamente (véase nuestro resumen en el presente monográfico, en la sección de mesas redondas). En este taller presentaremos con 
mayor detenimiento los antecedentes y particularidades sobre cómo preparamos a nuestros estudiantes de FIPEF para que lleguen a tener éxito como profesores de EF. Desarrollaremos el enfoque educativo y evaluativo de nuestro programa de FIFPEF y comentaremos ejemplos de tareas de aprendizaje que utilizamos en nuestro programa para formar a nuestros estudiantes. Presentaremos ejemplos específicos de programas de evaluación (i.e., prácticum, dominios de movimiento, teoría), tareas de evaluación auténticas (formativas y sumativas) y herramientas de evaluación (digitales). Durante el taller, y para lograr un aprendizaje mutuo, invitaremos a los participantes a discutir nuestro enfoque de la FIPEF y a compartirlo y compararlo con sus propias experiencias.

Palabras clave: Formación del profesorado de educación física; modelo 4C/DI; tareas de aprendizaje; evaluación; evaluación programática

\section{Resumo}

Nos últimos anos, o currículo da Formação de Professores de Educação Física (FPEF) da Fontys University tem sido completamente redesenhado (ver o nosso resumo nesta monografia, sessão de mesas redondas). Durante este workshop apresentaremos informação mais detalhada sobre o modo como nós preparamos os nossos estudantes da FPEF, de forma a tornarem-se professores de Educação Física bem-sucedidos. Vamos desenvolver as abordagens educacionais e de avaliação usadas no nosso programa FPEF e discutir exemplos de tarefas de avaliação usadas no nosso programa para ensinar os nossos alunos. Serão apresentados exemplos específicos dos programas de avaliação (ex: estágio curricular, domínio dos movimentos, teoria), tarefas de avaliação autênticas (formativa e sumativa) e ferramentas de avaliação (digital). Durante o workshop e de forma a aprendermos uns com os outros, convidamos todos os participantes a discutirem a nossa abordagem de FPEF, e também, a partilharem e compararem com as suas próprias experiências.

Keywords: Formação de professores de educação física; modelo 4C/DI; tarefas de aprendizagem; avaliação; avaliação programática 